\title{
Cost-effectiveness of diabetic retinopathy screening programs using telemedicine: a systematic review
}

Daniel Avidor ${ }^{1 *}$, Anat Loewenstein ${ }^{1,2}$, Michael Waisbourd $^{3}$ and Amir Nutman ${ }^{1,4}$

\begin{abstract}
Background: Diabetic retinopathy (DR) is a significant global public health and economic burden. DR accounts for approximately $15-17 \%$ of all cases of total blindness in the USA and Europe. Telemedicine is a new intervention for DR screening, however, there is not enough evidence to support its cost-effectiveness. The aim of this study is to review the most recent published literature on economic evaluations of telemedicine in DR screening and summarize the evidence on the cost-effectiveness of this technology.
\end{abstract}

Methods: A systematic search of PubMed, Embase and Google Scholar for relevant articles published between January 2010 and January 2020. Studies were included if they met the following criteria: (1) recruited subjects with either type 1, type 2 diabetes (2) evaluated telemedicine technology (3) patients underwent primary screening for DR (4) compared a telemedicine-based intervention with standard care (5) performed an economic evaluation or provided sufficient data for evaluating the cost-effectiveness of the technology used.

Results: Of 2238 articles screened, seven studies were included. Four of the studies were conducted in developed countries: The United States, Singapore and two studies in Canada. Three studies were conducted in developing countries: India, Brazil and South Africa. The patient populations in all studies were diabetic patients over the age of 18, previously not screened for DR. All seven studies used a telemedicine program which included capturing a retinal image and subsequently transmitting it to an ocular imaging center to assess the severity of DR. All studies compared telemedicine to a standard screening method for DR, including the option of no screening as standard of care. Although telemedicine requires initial and maintenance costs, it has the potential to provide significant cost savings by increasing patients' working ability, increasing independent living ability, increasing quality of life and reducing travel costs.

Conclusions: Diabetic retinopathy telemedicine technology has the potential to provide significant cost savings, especially in low-income populations and rural patients with high transportation costs.

Keywords: Diabetic retinopathy, Telemedicine screening, Teleophthalmology, Economic evaluation, Costeffectiveness

${ }^{*}$ Correspondence: Mirond88@gmail.com

${ }^{1}$ Sackler Faculty of Medicine, Tel-Aviv University (TAU), Tel-Aviv, Israel

Full list of author information is available at the end of the article

\begin{abstract}
Background
Diabetes mellitus (DM) incidence and prevalence is on the rise in recent years, causing significant morbidity and mortality [1]. In 2014, 422 million patients worldwide were diagnosed with DM, this number is expected to rise to 592 million by 2035, compared to 108 million in 1980 . In the European region, there are about 60 million people
\end{abstract}


with DM comprising $10.3 \%$ of men and $9.6 \%$ of women aged 25 years and over [2]. In the United States, there are 30.2 million people aged 18 and over with DM, which represent $12.2 \%$ of the entire population; $11.7 \%$ of the women and $12.7 \%$ of the men [3].

Diabetic retinopathy (DR) is a common microvascular complication of DM, which causes irreversible damage to the retina. The World Health Organization (WHO) has estimated that DR accounts for approximately $15-17 \%$ of all cases of total blindness in the USA and Europe [4]; it is the leading cause of blindness amongst the working-age population in developed countries [5]. More than 60\% of individuals with type $2 \mathrm{DM}$ and virtually all patients with type 1 DM develop DR within the first 20 years of diagnosis of the disease [6]. With the increasing prevalence of DM, the number of individuals with DR is also likely to rise. Globally, the number of people with DR is expected to grow from 126.6 million in 2010 to 191.0 million by 2030 , and some studies estimate that the number of patients with vision-threatening DR will increase from 37.3 to 56.3 million [7]. These disturbing numbers make DR a significant global public health and economic issue.

Multiple studies and clinical trials have reported the benefit of early detection and timely treatment in reducing the risk of vision loss from DR [8] and decreasing the global burden of blindness [9]. Active screening for DR is important because most patients who develop DR have no symptoms until the very late stages, and by then it is often too late for effective treatment. Although there are several ways to detect DR, the gold standard is a dilated fundus examination using an indirect ophthalmoscope or a slit lamp bio-microscope by an ophthalmologist. The American Academy of Ophthalmology recommends annual DR examinations for patients with type 1 and type $2 \mathrm{DM}$, however, in practice only $50 \%$ to $65 \%$ of patients receive this recommended screening [10].

Multiple patient barriers to DR screening exist, including poor access to care, lack of time, high out-of-pocket expenses, insufficient patient knowledge and awareness of DR, and lack of care coordination, especially among low-income populations, and ethnical minorities $[11,12]$. These barriers are further magnified among developing countries [11, 13].

Telemedicine is the exchange of medical data using electronic technology that allows a patient's medical case to be evaluated and monitored by a remotely located physician [11]. Telemedicine screening programs to assess DR disease through remote retinal imaging can effectively diagnose DR and recommend proper care interventions [14]. There is a variety of retinal imaging technologies, such as digital imaging systems, handheld fundus cameras, non-mydriatic cameras and the use of smartphones as a fundus camera [15]. The goal of telemedicine-teleophthalmology programs in DR is to increase the number of patients screened and monitor those at risk for progression [12]. Teleophthalmology screening programs have the potential to increase access to care in remote areas, save the patient time and travel costs, and to identify those who have the immediate need for retinal evaluation versus those who do not $[14,16]$.

Teleophthalmology can produce the same clinical outcomes as the traditional face to face examination [17]. Tan et al. [18] published a systematic review of teleophthalmology diagnostic accuracy compared with face to face consultation and found that teleophthalmology was considered superior to face to face consultation in one study and comparable in six studies. Surendran et al. [19] demonstrated that overall, the published medical literature agrees that teleophthalmology programs are accurate and safe alternatives to the traditional DR screening.

Although studies have examined the clinical benefits of telemedicine [16], the use of teleophthalmology is currently in its infancy and has yet to gain widespread acceptance, and only a few studies have assessed the economic benefits. The purpose of this systematic review is to summarize the data on economic evaluation and cost-effectiveness of DR screening in type 1 and 2 diabetes patients using teleophthalmology technology as compared to standard care.

\section{Methods}

The systematic review protocol was not registered in any database. A literature search was performed using PubMed, Embase and Google Scholar search engines for relevant articles published between January 2010 and January 2020. We used a combination of medical subject headings and text terms to generate four subsets of citations: one for diabetic retinopathy, the second for telemedicine, based on a search using the terms 'telemedicine,' 'telehealth', 'teleophthalmology' and 'teleretinal', the third used the term 'screening' and the fourth for economic analysis based on the terms 'cost-effectiveness', 'cost-utility', 'cost-benefit', 'incremental cost-effectiveness ratio', 'economic analysis' and 'quality of life years'. The terms were combined to generate a subset of citations relevant to the research question. Also, we examined the reference lists in original and review articles to identify additional studies that were not captured by the electronic searches. Articles in languages other than English and narrative reviews were excluded. We included studies if they met the following criteria: (1) recruited subjects with either type 1 or type 2 DM (2) evaluated DR telemedicine technology (3) patients underwent primary screening for DR (4) compared a telemedicinebased intervention with standard care (5) performed an economic evaluation or provided sufficient data for 
evaluating the cost-effectiveness of the technology used. Studies that included patients with known DR or patients with other co-morbid eye disease were excluded, as well as studies with no description of subjects. All identified studies were exported into the citation software package 'Mendeley' and duplicates were removed. We included only published data, which may lead to an inherent problem of publication bias.

\section{Results}

Of 2238 articles screened, 30 full-text articles were assessed for eligibility criteria, and seven studies were included in this review (Fig. 1).

Four of the studies were conducted in developed countries: The United States, Singapore and two studies in
Canada. Three studies were conducted in developing countries: India, South Africa and Brazil (Table 1). All studies reported the economic analyses using local currency, except for one. To ease the comparison between studies, we converted local currency to United States dollars (USD) based on the exchange rate at the time of writing this manuscript. The patient populations in all studies were diabetic patients over the age of 18 , without known DR.

All studies included used a telemedicine program which included capturing a retinal image and subsequently transmitting it to an ocular imaging center to assess the severity of DR. All studies compared telemedicine to a standard screening method for DR, including the option of no screening as the standard of care.

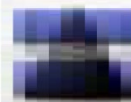

PRISMA 2009 Flow Diagram

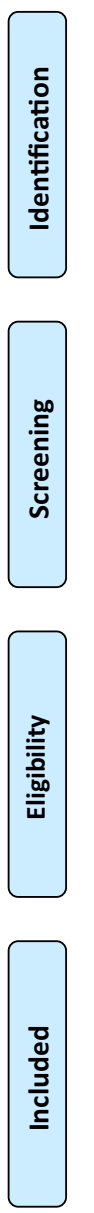

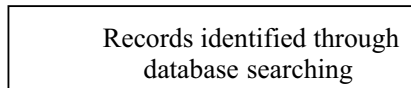
database searching $(\mathrm{n}=2238)$
Additional records identified through other sources $(\mathrm{n}=0)$

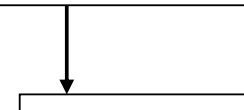

Records after duplicates removed $(\mathrm{n}=2229)$

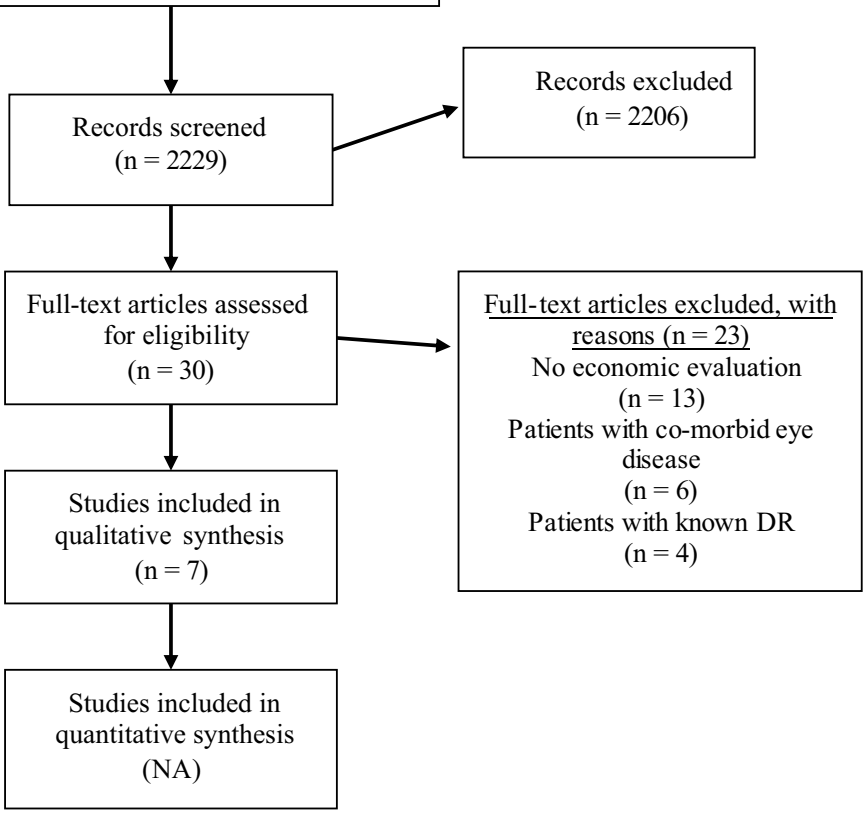

Fig. 1 Flow diagram of study selection. Depicts the flow of the studies included in this article. Maps out the number of studies identified, included and excluded, and the reasons for exclusions 


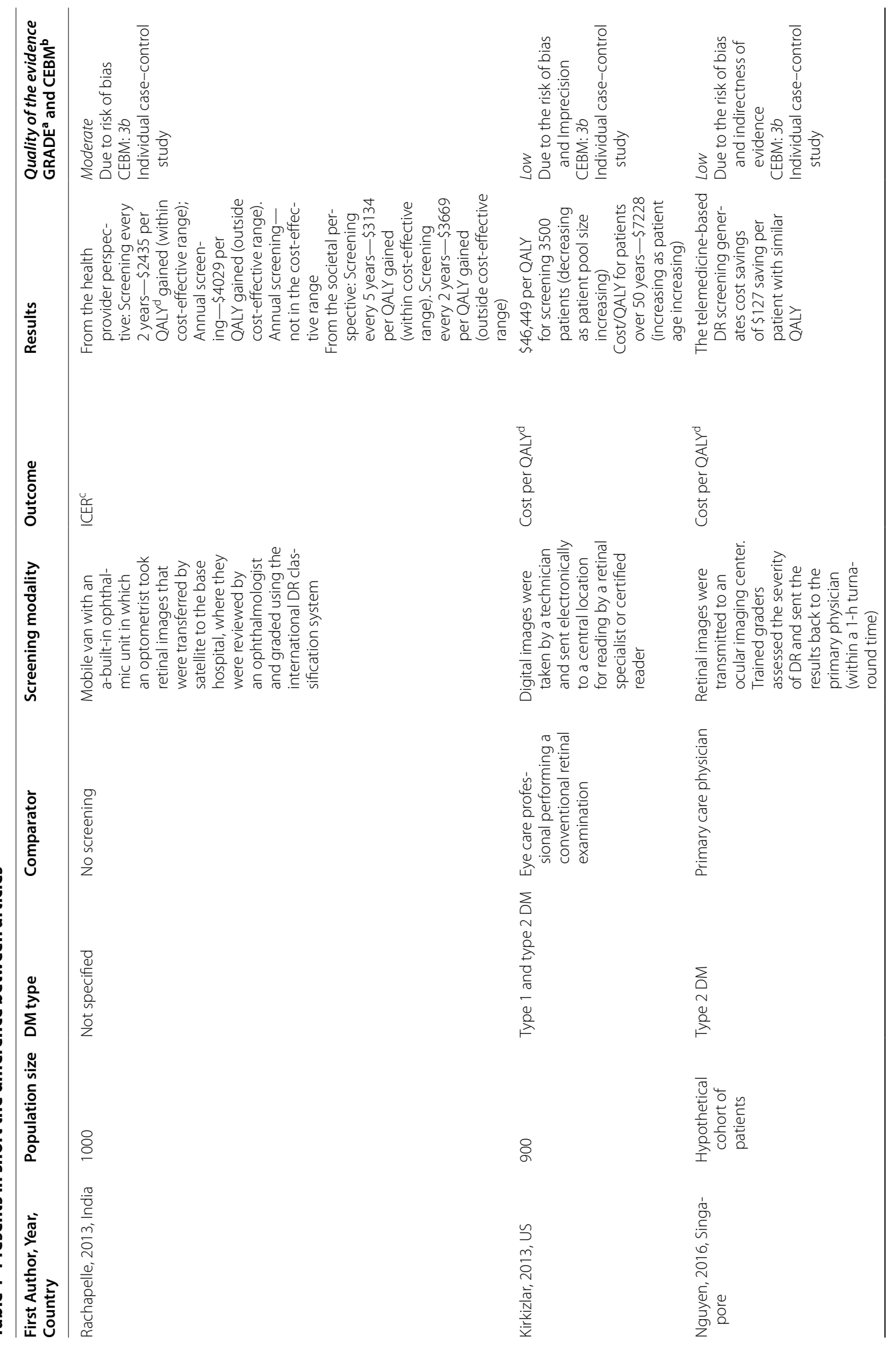




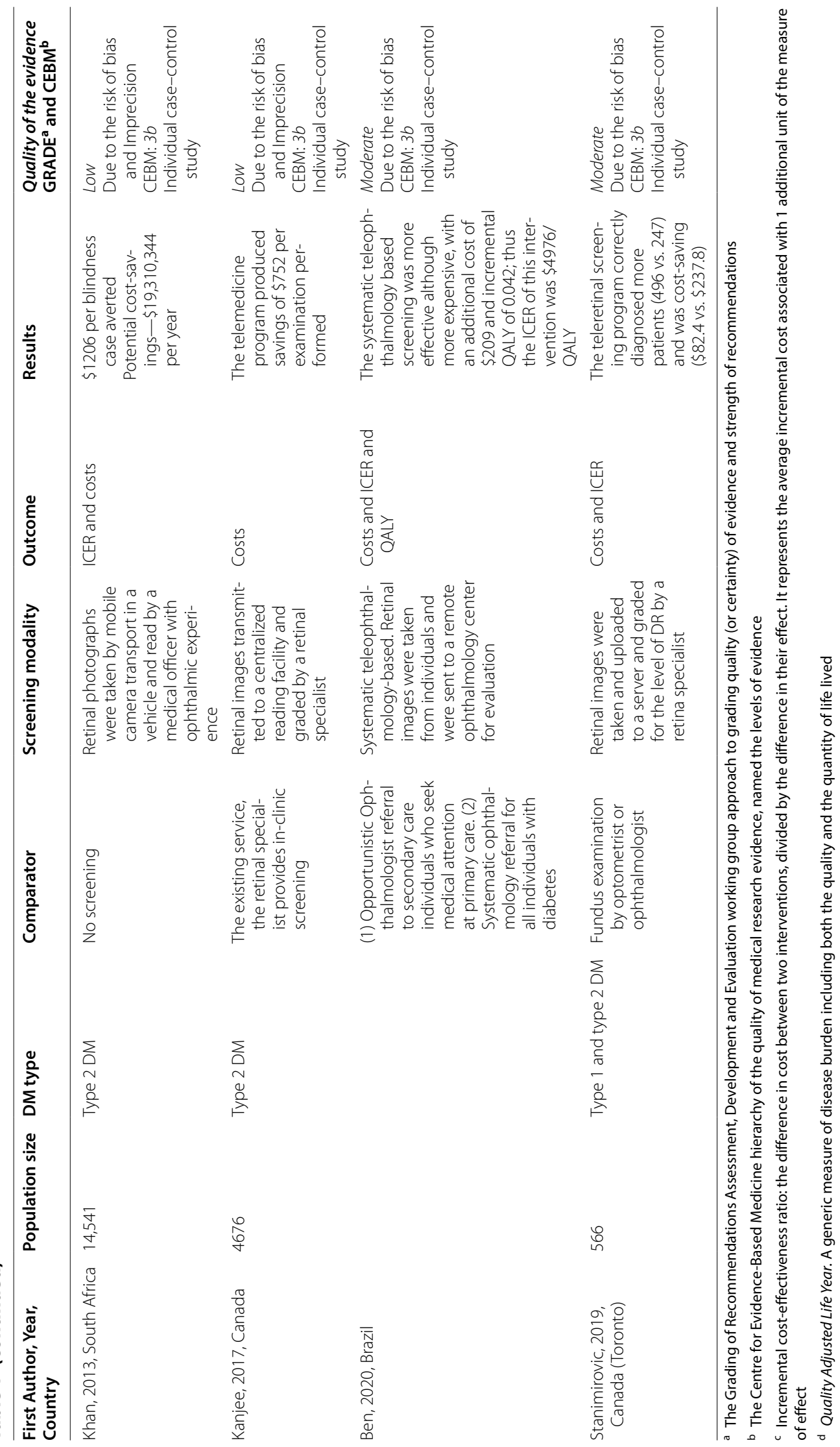


Kirkizlar et al. screened a total of 900 patients aged 18-99 years with type 1 and type 2 DM. The participants were from the Veterans Health Administration department in the USA and from different ethnic groups [20]. They used the economic model of Chapman et al. [21] to estimate the cost-utility of DR screening using telemedicine vs. conventional DR screening examination and considered cost-effectiveness if the cost per QALY of the program is less than an established monetary threshold of $\$ 50,000$. They demonstrated that population size and age determined the cost-effectiveness of the program. A telemedicine screening program is cost-effective if the screened population size is over 3500 people. For a population of 3500 patients included in a screening program, the average costs are $\$ 46,449$ per quality-adjusted life years (QALY) vs. $\$ 20,392$ per QALY for a population size of 9000. However, telemedicine screening for patients under the age of 50 years or older than 80 years was not cost-effective (Additional file 1: Table S1).

Rachapelle et al. [22] used a Markov model to estimate the cost-utility of a telemedicine screening program with different screening intervals in comparison with no screening, which was the current standard of care in India. The study was based on a hypothetical cohort of 1000 rural diabetic patients aged 40 years. The costs and outcomes were estimated for a period of 25 years. They estimated the costs per person from a healthcare provider perspective and societal perspective (the sum of the health provider and household costs). Utility was defined using the time trade-off method by interviews with diabetic patients; the maximum number of years the patient was willing to trade for perfect vision. The incremental cost-effectiveness ratio (ICER) threshold used to determine cost-effectiveness was $<\$ 3183$ per QALY gained, using WHO recommendations. Their cost-utility model suggested that from a health provider's perspective, the cost of telemedicine screening once every 2 years was $\$ 2435$ per QALY gained, which is cost-effective compared to no DR screening for rural diabetic patients. Annual screening fell outside of the cost-effective range, $\$ 4029$ per QALY gained, however, with $85 \%$ of the additional costs attributable to hospital fees. From a societal perspective, the cost of screening every 5 years was $\$ 3134$ per QALY gained, which was considered cost-effective. However, the cost of screening every 2 years at $\$ 3669$ per QALY gained, was not considered cost-effective.

Nguyen et al. [23] used a Markov model to estimate the cost-effectiveness from the health system and societal (medical and non-medical costs) perspectives. They used the UK National Institute for Health and Care Excellence (NICE)'s ICER threshold of $£ 30,000$ per QALY to determine cost-effectiveness. The study population consisted of a hypothetical cohort of patients with type $2 \mathrm{DM}$. The mean age was 55 years, which reflected the actual age distribution of the Singaporean population of patients with type $2 \mathrm{DM}$. They reported that over a lifetime telemedicine was cost-saving, from both the health system and societal perspectives, compared with the current practice, a family physician eye examination, with similar QALYs across the two programs. The main savings were from the societal perspective; every patient with DM who used telemedicine would generate cost savings of approximately \$127 USD.

Khan et al. [24] analyzed the cost-effectiveness of a telemedicine screening program in South Africa. Patients were seen in three community health centers and all of them were from low social-economic backgrounds. All patients had type $2 \mathrm{DM}$. The analysis included only direct medical costs, as well as transportation costs. The comparator was defined as the current practice, where people with DM were not screened for DR. The outcome measure was cost per one blindness case averted. They calculated that the cost for telemedicine screening was $\$ 22$ per person and the ICER was $\$ 1206$ per blindness case averted; less than the threshold used which was the annual disability grant in South Africa (\$1393). A telemedicine DR screening program would be cost-effective even if just $65 \%$ of people with DM were screened. The main limitation of this study was that it took place in Cape Town, which is different from other parts of South Africa as; DR prevalence and the stage at which patients present to health care are lower than other parts of South Africa. Additionally, costs will initially increase in areas that have a significantly high prevalence of DM, but savings will occur in the long term.

Kanjee et al. [25] estimated the costs of a telemedicine program in a Manitoban cohort. They performed a retrospective chart analysis of 4676 patients from the Manitoba Retinal Screening Vision Program. Patients were included if they were 18 years or older and had a diagnosis of type $2 \mathrm{DM}$. They demonstrated a lower cost for telemedicine compared with conventional in-clinic screening. On average, the telemedicine program produced savings of $\$ 752$ per examination performed, compared to in-clinic screening.

Ben et al. [26] performed a model-based economic evaluation to compare three DR screening practices in Brazil. The population included were patients with type 2 DM aged 40 years and without known DR. Analyses were performed from the Healthcare System perspective. The three DR screening strategies were: (1) the common practice, opportunistic ophthalmology referral, i.e. ophthalmologist referral for individuals who seek medical attention at primary care (ophthalmologist referral is covered by the public primary care program in Brazil). (2) Systematic ophthalmologist referral, i.e. ophthalmologist 
referral for all individuals with type 2 DM (3) systematic teleophthalmology referral where retinal images were sent to a remote ophthalmology center for evaluation. The ICER threshold was determined based on the GDP per capita for Brazil. Interventions with an ICER less than $\$ 10,382 /$ QALY were considered cost effective. The systematic teleophthalmology based screening was more effective although more expensive: an additional cost of $\$ 209$ and incremental QALY of 0.042; thus the ICER of this intervention was $\$ 4976 /$ QALY, which is under the ICER threshold (\$14,953/QALY).

Stanimirovic et al. [27] assessed the cost-effectiveness of a pilot Toronto teleophthalmology screening program using a decision tree model. Images were taken by the primary care provider and analyzed by a retina specialist. A total of 566 patients aged $>20$ years, with both type 1 and $2 \mathrm{DM}$ were screened. The economic analysis was conducted from a health care perspective and the cost-effectiveness of teleophthalmology screening was assessed as cost per case detected (true-positive) and cost per case correctly diagnosed (true-positive and true-negative). Compared to conventional screening by a primary care eye specialist, the teleophthalmology screening program correctly diagnosed more patients (496 vs. 247 ) and was cost-saving ( $\$ 82.4$ vs. $\$ 237.8$ ). Thus, the teleophthalmology program was the dominant strategy $($ ICER $<0)$.

\section{Discussion}

Early detection, accurate diagnosis, and timely treatment of DR have long been established as a means to significantly reduce vision loss from DM and improve public health [15]. Multiple professional organizations, including the American Academy of Ophthalmology, recommend annual retinal examinations for patients with DM [10]. Despite these recommendations and the known fact that screening for DR is a cost-effective method to reduce blindness [24], many DM patients do not receive recommended screening. In the United States and other developed countries, only half of the diagnosed population with DM is screened annually for DR [28], therefore diabetes-related vision loss remains the major cause of blindness in western populations [15]. We can only assume that in developing countries the proportion of screened patients is likely even lower. The reasons for poor compliance are several, including lack of patient education, lack of access to care, and geographic limitations [12]. Gibson et al. [29] demonstrated that $73 \%$ of patients with DR unaware of their condition.

Telemedicine can overcome geographical, financial, and socioeconomic barriers. Blomdahl et al. [30] reported that teleophthalmology is particularly useful when the distance to an ophthalmologist is an obstacle to diagnosis and treatment. Teleophthalmology can expand standards of eye care delivery by extending access to care, offering alternative methods for receiving appropriate care, and integrating DM eye care into the patient's total healthcare. A high satisfaction level and acceptance from patients is reported in the majority of the studies because of increased accessibility and reduced traveling cost and time [17].

In this study, we evaluated the cost-effectiveness of teleophthalmology programs reported in a variety of settings, including the United States, Canada, Singapore, India, Brazil, and South Africa. In all studies teleophthalmology was cost-effective based on the results of the economic evaluations. Kirkizlar et al. [20] demonstrated that a teleophthalmology screening program is costeffective for a population size of over 3500 patients aged 50-80 years in the US. Rachapelle et al. [22] reported that telemedicine screening programs are cost-effective compared with no DR screening for Indian rural diabetic patients. Khan et al. [24] demonstrated that teleophthalmology is a cost-effective screening tool for DR in a South African population, even if merely $65 \%$ of people with DM are screened. Nguyen et al. [23] reported that every patient with DM who uses the telemedicine program generates cost savings of $\$ 127$ over a lifetime. Currently, there are 170,000 DM patients in Singapore, thus the expected total savings if a teleophthalmology screening program would be implemented is estimated to be $\$ 21.6$ million $(170,000 \times \$ 127)$. Kanjee et al. [25] demonstrated a lower cost to the telemedicine program compared to the conventional in-clinic screening in a Canadian cohort. Ben et al. [26] presented that systematic teleophthalmology based screening is cost-effective compared to opportunistic ophthalmology-referral based screening in Brazil. Stanimirovic et al. [27] found that teleophthalmology screening dominated the standard of care in a Canadian pilot study (ICER $<0$ ).

The findings of this systematic review should be interpreted with caution due to the lack of data from randomized controlled trials (RCTs) and high risk of bias in the available data from observational studies. Another limitation is that DR screening was performed in each study by different health care professionals such as optometrists, general physicians, and clinical photographers and using different modalities, which makes it difficult to synthesize results.

Although telemedicine requires initial and maintenance costs, we conclude from this review that this intervention has the potential to provide significant cost savings via accurate DR diagnosis and treatment, thus increasing patients working ability, increasing independent living ability, increasing quality of life and reducing travel costs, and are cost-effective from both the healthcare and a societal perspectives. These programs are even 
more cost-effective in low-income populations and rural diabetic patients with high transportation costs [31-33]. With advances in technology which include better and faster telecommunication, cloud storage, miniaturization of equipment (smartphones with digital cameras) and automation of retinal image analysis, teleophthalmology screening programs can be optimized. This optimization includes improving productivity, safety standards, quality assurance, and sustainability, thus improving patient care and long-term outcomes [11, 34]. As the costs of equipment are constantly reduced, teleophthalmology services costs are reduced, thus improving cost-effectiveness.

\section{Conclusion}

Teleophthalmology is an emerging technology with the potential to improve accessibility to DR screening programs and positively influence patient care on a large scale. This intervention has the potential to improve compliance with DR screening and reduce the incidence of vision-threatening complications of diabetes, thus increasing patients' working ability, independent living ability and quality of life, as well as reduce costs such as traveling costs and physician time. Although our review identified only a few economic evaluations of screening DR with telemedicine programs to date, these studies demonstrated cost-effectiveness. We propose that more robust RCTs will be conducted to evaluate the application of teleophthalmology to monitor DR and other eye conditions. In the future, with the aid of automated systems for grading DR using artificial intelligence (AI), this technology has the potential to be even more accurate, save physician specialist time, and improve the costeffectiveness of the DR screening service.

\section{Supplementary information}

Supplementary information accompanies this paper at https://doi. org/10.1186/s12962-020-00211-1.

Additional file 1: Table S1. Table of full text articles excluded with reasons.

\section{Abbreviations}

DR: Diabetic retinopathy; DM: Diabetes mellitus; ICER: Incremental cost-effectiveness ratio; QALY: Quality-adjusted life years.

\section{Acknowledgements}

Not applicable.

\section{Authors' contributions}

DA data collection and analysis; AN and DA project design, data interpretation, writing, and editing of manuscript; AL and MW have drafted the work, substantively revised it and were major contributors in writing the manuscript. All authors read and approved the final manuscript.

\section{Funding}

No funding was obtained for this study.
Availability of data and materials

All data generated or analyzed during this study are included in this published article.

Ethics approval and consent to participate

Not applicable.

Consent for publication

Not applicable.

\section{Competing interests}

None of the authors have any competing interests.

\section{Author details}

${ }^{1}$ Sackler Faculty of Medicine, Tel-Aviv University (TAU), Tel-Aviv, Israel. ${ }^{2}$ Ophthalmology Department, Tel-Aviv Sourasky Medical Center, Tel-Aviv, Israel.

${ }^{3}$ Glaucoma Research Center, Tel-Aviv Sourasky Medical Center, Tel-Aviv, Israel.

${ }^{4}$ Tel-Aviv Sourasky Medical Center and National Center for Infection Control,

Tel-Aviv, Israel.

Received: 30 August 2019 Accepted: 26 March 2020

Published online: 06 April 2020

\section{References}

1. Zimmet P, Alberti KGMM, Shaw J. Global and societal implications of the diabetes epidemic. Nature. 2001;414(6865):782-7.

2. $\mathrm{WHO}$ progress report on the implementation of the European Charter on Counteracting Obesity. 2018. http://www.euro.who.int/en/about-us/ regional-director/speeches-and-presentations-by-year/2010/who-progr ess-report-on-the-implementation-of-the-european-charter-on-count eracting-obesity. Accessed 23 July 2018.

3. For Disease Control C. National Diabetes Statistics Report, 2017 estimates of diabetes and its burden in the United States background. 2017. https ://www.cdc.gov/diabetes/pdfs/data/statistics/national-diabetes-statistics -report.pdf. Accessed 28 Aug 2018.

4. Resnikoff S, Pascolini D, Etyaale D, Kocur I, Pararajasegaram R, Pokharel GP, et al. Global data on visual impairment in the year 2002. Bull World Health Org. 2004;82:844-51.

5. Porta M, Bandello F. Diabetic retinopathy. Diabetologia. 2002;45(12):161734. https://doi.org/10.1007/s00125-002-0990-7.

6. Barricks M, Barricks M. The wisconsin epidemiologic study of diabetic retinopathy. Arch Ophthalmol. 1995;113(6):702. https://doi.org/10.1001/ archopht.1995.01100060024015.

7. Congdon N, Zheng Y, He M. The worldwide epidemic of diabetic retinopathy. Indian J Ophthalmol. 2012;60(5):428.

8. Saaddine JB, Honeycutt AA, Narayan KMV, Zhang X, Klein R, Boyle JP. Projection of diabetic retinopathy and other major eye diseases among people with diabetes mellitus. Arch Ophthalmol. 2008;126(12):1740. https ://doi.org/10.1001/archopht.126.12.1740.

9. Shi L, Wu H, Dong J, Jiang K, Lu X, Shi J. Telemedicine for detecting diabetic retinopathy: a systematic review and meta-analysis. $\mathrm{Br} J$ Ophthalmol. 2015;99(6):823-31.

10. Schoenfeld ER, Greene JM, Wu SY, Leske MC. Patterns of adherence to diabetes vision care guidelines: baseline findings from the Diabetic Retinopathy Awareness Program. Ophthalmology. 2001;108(3):563-71.

11. Das T, Raman R, Ramasamy K, Rani PK. Telemedicine in diabetic retinopathy: current status and future directions. Middle East Afr J Ophthalmol. 2015;22(2):174-8.

12. Rathi S, Tsui E, Mehta N, Zahid S, Schuman JS. The current state of teleophthalmology in the United States. Ophthalmology. 2017;124(12):1729-34.

13. Gupta A, Cavallerano J, Sun JK, Silva PS. Evidence for telemedicine for diabetic retinal disease. Semin Ophthalmol. 2017;32(1):22-8. https://doi. org/10.1080/08820538.2016.1228403.

14. Gupta A, Cavallerano J, Sun JK, Silva PS. Evidence for telemedicine for diabetic retinal disease. In: Seminars in ophthalmology. 2017.

15. Zimmer-Galler IE, Kimura AE, Gupta S. Diabetic retinopathy screening and the use of telemedicine. Curr Opin Ophthalmol. 2015;26(3):167-72. 
16. Hailey D, Roine R, Ohinmaa A. Systematic review of evidence for the benefits of telemedicine. J Telemed Telecare. 2002. https://doi. org/10.1258/1357633021937604.

17. Sreelatha OK, Ramesh SV. Teleophthalmology: improving patient outcomes? Clin Ophthalmol. 2016;10:285-95.

18. Tan IJ, Dobson LP, Bartnik S, Muir J, Turner AW. Real-time teleophthalmology versus face-to-face consultation: a systematic review. J Telemed Telecare. 2017. https://doi.org/10.1177/1357633X16660640.

19. Surendran TS, Raman R. Teleophthalmology in diabetic retinopathy. J Diabetes Sci Technol. 2014;8(2):262-6. https://doi.org/10.1177/19322 96814522806.

20. Kirkizlar E, Serban N, Sisson JA, Swann JL, Barnes CS, Williams MD. Evaluation of telemedicine for screening of diabetic retinopathy in the veterans health administration. Ophthalmology. 2013;120(12):2604-10.

21. Chapman RH, Berger M, Weinstein MC, Weeks JC, Goldie S, Neumann PJ. When does quality-adjusting life-years matter in cost-effectiveness analysis? Health Econ. 2004;13(5):429-36. https://doi.org/10.1002/hec.853.

22. Rachapelle $S$, Legood R, Alavi Y, Lindfield R, Sharma T, Kuper H, et al. The cost-utility of telemedicine to screen for diabetic retinopathy in india. Ophthalmology. 2013;120(3):566-73. https://doi.org/10.1016/j.ophth a.2012.09.002

23. Nguyen HV, Tan GSW, Tapp RJ, Mital S, Ting DSW, Wong HT, et al. Costeffectiveness of a national telemedicine diabetic retinopathy screening program in Singapore. Ophthalmology. 2016;123(12):2571-80. https:// doi.org/10.1016/j.ophtha.2016.08.021.

24. Khan T, Bertram MY, Jina R, Mash B, Levitt N, Hofman K. Preventing diabetes blindness: cost effectiveness of a screening programme using digital non-mydriatic fundus photography for diabetic retinopathy in a primary health care setting in South Africa. Diabetes Res Clin Pract. 2013;101(2):170-6. https://doi.org/10.1016/j.diabres.2013.05.006.

25. Kanjee R, Dookeran RI, Mathen MK, StockI FA, Leicht R. Six-year prevalence and incidence of diabetic retinopathy and cost-effectiveness of tele-ophthalmology in Manitoba. Can J Ophthalmol. 2017;52(6):S15-8. https://doi.org/10.1016/j.jcjo.2016.05.002.

26. Ben ÂJ, Neyeloff JL, De Souza CF, Rosses APO, De Araujo AL, et al. Costutility analysis of opportunistic and systematic diabetic retinopathy screening strategies from the perspective of the Brazilian public healthcare system key points for decision-makers. Appl Health Econ Health Policy. 2020;18:57-68. https://doi.org/10.1007/s40258-019-00528-w.

27. Stanimirovic A, Francis T, Shahid N, Sutakovic O, Merritt R, Brent MH, et al. Tele-retina screening of diabetic retinopathy among at-risk populations: an economic analysis. Can J Ophthalmol. 2019;55:8-13.

28. Services H, Mathews Burwell S. The Department of Health and Human Services 2014 annual report on the quality of health care for adults enrolled in medicaid. 2014. https://www.medicaid.gov/medicaid/quali ty-of-care/downloads/2014-adult-sec-rept.pdf. Accessed 29 July 2018.

29. Gibson DM. Diabetic retinopathy and age-related macular degeneration in the U.S. Am J Prev Med. 2012;43(1):48-54.

30. Blomdahl S, Marén N, Lof R. Tele-ophthalmology for the treatment in primary care of disorders in the anterior part of the eye. J Telemed Telecare. 2001;7(1_suppl):25-6. https://doi.org/10.1177/1357633×010070s110.

31. Choremis J, Chow DR. Use of telemedicine in screening for diabetic retinopathy. Can J Ophthalmol. 2003;38(7):575-9.

32. Fonda SJ, Bursell S-E, Lewis DG, Garren J, Hock K, Cavallerano J. The relationship of a diabetes telehealth eye care program to standard eye care and change in diabetes health outcomes. Telemed e-Health. 2007;13(6):635-44. https://doi.org/10.1089/tmj.2007.0025.

33. Wei JC, Valentino DJ, Bell DS, Baker RS. A Web-based telemedicine system for diabetic retinopathy screening using digital fundus photography. Telemed e-Health. 2006;12(1):50-7. https://doi.org/10.1089/tmj.2006.12.50.

34. DeBuc DC. The role of retinal imaging and portable screening devices in tele-ophthalmology applications for diabetic retinopathy management. Curr Diabetes Rep. 2016;16(12):132. https://doi.org/10.1007/s1189 2-016-0827-2.

\section{Publisher's Note}

Springer Nature remains neutral with regard to jurisdictional claims in published maps and institutional affiliations.
Ready to submit your research? Choose BMC and benefit from:

- fast, convenient online submission

- thorough peer review by experienced researchers in your field

- rapid publication on acceptance

- support for research data, including large and complex data types

- gold Open Access which fosters wider collaboration and increased citations

- maximum visibility for your research: over $100 \mathrm{M}$ website views per year

At BMC, research is always in progress.

Learn more biomedcentral.com/submissions 